\title{
Relationship between Aortic Valve Sclerosis and the Severity of Coronary Artery Disease in Patients Undergoing Diagnostic Coronary Angiography
}

\section{Abstract}

Objective: Mitral annulus calcification (MAC) and aortic valve sclerosis (AVS) represent a degenerative process that progresses with advancing age. These calcifications are associated with coronary risk factors. Thus, they are also considered as a manifestation of generalized atherosclerosis in elderly populations. Aortic valve sclerosis (AVS) is defined as calcification and thickening of the aortic valve in the absence of obstruction of ventricular outflow [1]. Atherosclerosis risk factors and clinical cardiovascular disease are independently associated with AVS, suggesting that AVS represents an atherosclerosis-like process involving the aortic valve [2]. We sought to determine the association between AVS and the extent of coronary atherosclerosis by means of the Gensini score system, which was calculated to yield a measure of the extent and severity of coronary atherosclerosis in patients who had suspected or known ischemic heart disease and who were referred for coronary angiography. The aim of this study was to investigate the association between aortic valve sclerosis and mitral annulus calcification with severity of coronary atherosclerosis measured using Gensini score.

Patients and methods: The study population consisted of 200 Egyptian patients who were underwent coronary angiography for suspected or known coronary atherosclerosis. After taking a detailed clinical history, with special attention to risk factor identification, all patients underwent complete transthoracic echocardiographic examination before they underwent coronary angiography on different days. Each of the tests was interpreted by two observers who were unaware of the results of the other tests. According to Tolstrup et al. [3] five grades (from grade 0 to grade 4) for AVS were classified. Grades 0 and 1 were classified as normal, while grades 2-4 were classified as AVS.

Gensini score was calculated after the coronary angiography to determine the severity of coronary artery disease. And then patients were divided in to two groups according to Transthoracic Echocardiographic examination.

Group 1: Normal aortic valve group (Grades 0 - 1).

Group 2: Aortic sclerosis group (Grades 2 -4).

Results: The study subjects were divided into two main groups according to presence and absence of aortic valve sclerosis as follow:

Group I: Includes 75 patients with normal aortic valve.

Group II: Includes 125 patients with aortic valve sclerosis.

The group (II) was further subdivided into two subgroups according to

\section{Introduction}

Aortic valve sclerosis (AVS) is defined as calcification and thickening of the aortic valve in the absence of obstruction of ventricular outflow. Asymptomatic AVS, identified by echocardiography in the absence of outflow obstruction, is common in the elderly and affects $21-26 \%$ of adults over 65 years of age [2,4]. Atherosclerosis risk factors and clinical cardiovascular diseases are independently associated with AVS, suggesting that AVS represents an atherosclerosis-like process

Research Article
Volume 11 Issue 1 - 2018
Peter Zohair Halliem Khilla1 ${ }^{*}$, Osama Sanad ${ }^{2}$,
Tarek Abo El Azm ${ }^{3}$ and Ahmed Ramzy
${ }^{1}$ Faculty of Medicine, Benha University, Egypt
${ }^{2}$ Professor of Cardiology, Benha Faculty of Medicine, Egypt
${ }^{3}$ Assistant Professor of Cardiology, Benha Faculty of Medicine,
Egypt
${ }^{4}$ Lecturer of Cardiology, Benha Faculty of Medicine, Egypt
*Corresponding author: Peter Zohair Haliem Khilla, Faculty
of Medicine, Benha University, Egypt,
Email: peterkhilla21@yahoo.com
Received: December 11, 2017 | Published: January 19,
2018

the degree of aortic valve sclerosis as follow:

Group IIa: Includes 85 patients with mildly accentuated reflectance, thickness $2-4 \mathrm{~mm}$ of the aortic valve leaflets.

Group IIb: Includes 40 patients with generalized hyper-reflectance, thickness $>4 \mathrm{~mm}$ of the aortic valve leaflets.

Gensini score was found to be significantly higher in the sclerotic group $(42.05 \pm 33.9)$ compared to the normal group $(14.21 \pm 21.69)$ ( $p$ value $<$ $0.001)$.

Also Gensini score was found to be of statistically significant different between the two patient subgroups compared to the control group ( $p$ value $<0.001$ )

Conclusion: Aortic valve sclerosis (AVS) is strongly correlated with the extent of coronary artery disease and may predict it. Echocardiographic detection of AVS in patients undergoing coronary angiography may be applied as a new surrogate marker of the extent of coronary atherosclerosis. AVS is associated with atherosclerosis risk factors like age, dyslipidemia and diabetes mellitus.

Keywords: Aortic valve sclerosis (AVS); Coronary artery disease (CAD); Gensini score

involving the aortic valve [5-7]. Initial preliminary clinical and experimental studies suggest that AVS is a manifestation of the atherosclerotic process [8].

An association between AVS and the number of coronary vessels involved has recently been reported in patients with suspected ischemic heart disease [9]. We sought to determine the association between AVS and the extent of coronary atherosclerosis by means of the Gensini score system, which was calculated to yield a measure of the extent and severity of coronary 
atherosclerosis in patients who had suspected or known ischemic heart disease and who were referred for coronary angiography.

\section{Patients and Methods}

Selected two hundred patients with risk of coronary artery disease, referred to our Cath-lab in Cardiology Department, Benha University Hospital, and Mabara Misr El Kadima hospital for diagnostic coronary angiography for evaluation of coronary artery disease between May 2016 and May 2017 were included in this study.

Patients were selected according to the following:

\section{Inclusion criteria:}

Age: Less than 65 years

Patients with chronic stable angina indicated for coronary angiography according to ACC/AHA guidelines [10]:

\section{A. Class I}

a) CCS class III and IV angina on medical treatment. (Level of Evidence: B)

b) High-risk criteria on noninvasive testing regardless of anginal severity (Table $1 \Uparrow$ ). (Level of Evidence: A)

c) Patients who have been successfully resuscitated from sudden cardiac death or have sustained (>30 seconds) monomorphic ventricular tachycardia or nonsustained ( $<30$ seconds) polymorphic ventricular tachycardia. (Level of Evidence: B)

\section{B. Class IIa}

a) CCS class III or IV angina, which improves to class I or II with medical therapy. (Level of Evidence: C)

b) Serial noninvasive testing with identical testing protocols, at the same level of medical therapy, showing progressively worsening abnormalities. (Level of Evidence: $\mathrm{C}$ )

c) Patients with angina and suspected coronary disease who, due to disability, illness, or physical challenge, cannot be adequately risk stratified by other means. (Level of Evidence: C)

d) CCS class I or II angina with intolerance to adequate medical therapy or with failure to respond, or patients who have recurrence of symptoms during adequate medical therapy as defined above. (Level of Evidence: $\mathrm{C}$ )

e) Individuals whose occupation involves the safety of others (eg, pilots, bus drivers, etc) who have abnormal but not high-risk stress test results or multiple clinical features that suggest high risk. (Level of Evidence: C)

\section{Class IIb}

a) CCS class I or II angina with demonstrable ischemia but no high-risk criteria on noninvasive testing. (Level of Evidence: C)

b) Asymptomatic man or postmenopausal woman without known coronary heart disease with $\geq 2$ major clinical risk factors and abnormal but not high-risk criteria on noninvasive testing (performed for indications stated in the ACC/AHA noninvasive testing guidelines). (Level of Evidence: $\mathrm{C}$ )

c) Asymptomatic patients with prior MI with normal resting left ventricular function and ischemia on noninvasive testing but without high-risk criteria. (Level of Evidence: C)

d) Periodic evaluation after cardiac transplantation. (Level of Evidence: C)

e) Candidate for liver, lung, or renal transplant $\geq 40$ years old as part of evaluation for transplantation. (Level of Evidence: C)

\section{Exclusion criteria:}

a) Patients with aortic stenosis (transaortic flow velocity $>2.5$ $\mathrm{m} / \mathrm{s}$ )

b) Rheumatic valvular disease

c) Bicuspid aortic valve

d) Congenital heart disease

e) Hypertrophic obstructive cardiomyopathy

f) Poor acoustic windows

g) Patients on hemodialysis

h) Patients above 65 years old

\section{Methods}

*Informed consent

*Evaluation of patients:

\section{History taking}

A thorough history was taken from all members of the study group with special emphasis on:-

a. History of hypertension, (Systemic hypertension was defined as a systolic blood pressure greater than or equal to 130 $\mathrm{mmHg}$ and/or a diastolic pressure greater than or equal to $80 \mathrm{mmHg}$ and/or the use of anti-hypertensive medications) (11).

b. History of diabetes mellitus (Defined by hyperglycemia requiring previous or ongoing pharmacologic therapy).

c. History of ischemic heart disease (UA, MI, PTCA, CABG).

d. History of smoking (Smokers were defined as those with a current or recent history of smoking within the past 1 year).

\section{Clinical evaluation}

All patients were assessed clinically, including the evaluation of cardiovascular risk factors

\section{Electrocardiographic (ECG) evaluation}

12 leads electrocardiography were done to all patients for:

i. ST segment shift. 

ii. Reversal of T wave polarity.
iii. Pathological Q wave
iv. Chamber enlargement

Laboratory assessment:

i. Including fasting blood sugar, serum creatinine and serum cholesterol level.

\section{Transthoracic Echocardiography}

A Refurbished GE Vivid 7 Ultrasound Machine was used with transducer $2.5 \mathrm{MHz}$ All the patients were examined in the left lateral decubitus position. Echocardiographic images were acquired from the standard views (parasternal long-axis, parasternal short axis at level of the great vessels, apical fourchambers, apical five-chambers and apical two-chambers). Recordings and calculations of different cardiac chambers and ejection fractions were made according to the recommendations of the American Society of Echocardiography [12].

\section{Aortic valve assessment}

Two-dimensional echocardiographic assessment of the aortic valve was performed from the parasternal long- and short-axis -at level of the root of great vessels- view. Echo-Doppler assessment of aortic valve was performed in apical 5 chambers view. AVS was defined by focal areas of increased echogenicity and thickening of the aortic valve leaflets without restriction of leaflet motion and with a velocity of $<2.5 \mathrm{~m} / \mathrm{s}$ across the aortic valve (at least one abnormal leaflet per valve). According to Tolstrup et al. [3], five grades (from grade 0 to grade 4 ) for AVS were classified:

Grade 0: no thickening of the aortic valve leaflets, normal reflectance;

Grade 1: slightly accentuated reflectance, thickness $\leq 2 \mathrm{~mm}$;

Grade 2: mildly accentuated reflectance, thickness 2-4 mm;

Grade 3: generalized hyper-reflectance, thickness $\geq 4 \mathrm{~mm}$;

Grade 4: markedly hyper-reflectant masses, thickness > $6 \mathrm{~mm}$.

Grades 0 and 1 were included in group I since in our study intra- and inter-observer variability in these patients was not acceptable.

The location and extent of AVS were defined. Also, the number of affected cusps was recorded. According to Tolstrup et al. [3], four morphologic types of AVS were identified:

Type I: localized, non-nodular sclerosis.

Type II: localized, nodular sclerosis

Type III: diffuse sclerosis.

Type IV: mixed sclerosis.

All studies were recorded on digital memory and evaluated independently by two cardiologists with expertise in echocardiography.

Observer variability was determined by the reassessment of the 10 randomly selected subjects. There was no intra- or interobserver variability for the assessment of the presence or absence of the AVS. The observers who made the diagnosis of AVS had no knowledge of the clinical and angiographic results.

The study subjects were divided into two main groups:

Group I: Patients with normal aortic valve (grade 0-1).

Group II: Patients with aortic valve sclerosis (grade 2-4).

Group II was subdivided into two subgroups:

Group IIa: grade 2

Group IIb: grade 3-4

\section{Coronary angiography:}

Diagnostic coronary artery catheterizations were done to all patients to assess the severity and the extent of CAD. Coronary angiography was performed by the through femoral artery access. Evaluation of all coronary angiograms was made by two observers. Significant CAD on coronary angiograms was defined as stenosis of $50 \%$ or greater narrowing of the diameter in coronary arteries. The Gensini score was used to give a measure of severity of coronary atherosclerosis (Figure 1).

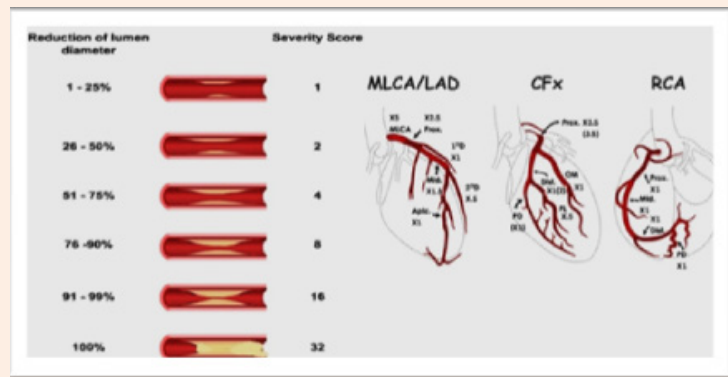

Figure 1: Gensini score.

a)point for $1-25 \%$ narrowing,

b) for $26-50 \%$ narrowing,

c) 4 for $51-75 \%$ narrowing,

d) 8 for 76-90\% narrowing,

e)16 for 91-99\% narrowing, and

f)32 for total occlusion.

This score is then multiplied by a factor that takes into account the importance of the lesion's position in the coronary arterial tree:

a) 5 for the left main coronary artery,

b) 2.5 for the proximal left anterior descending coronary artery and proximal left circumflex coronary artery (3.5 if left circumflex coronary artery is dominant),

c) 1.5 for the mid-region of the left anterior descending coronary artery, 
d) 1 for the distal left anterior descending coronary artery, the first diagonal, the proximal, mid and distal-region of the right coronary artery, the postero-descending, the mid- and distal-region of the left circumflex coronary artery ( 2 for both of them if left circumflex coronary artery is dominant) and the obtuse margin, and 0.5 For the second diagonal and the postero-lateral branch.

e) The Gensini score was expressed as the sum of the scores for the all coronary arteries [13].

\section{Statistical analysis}

All collected data were tabulated and analyzed by the appropriate statistical tests using SPSS version 17.0. Categorical data were described on numbers (percentage) and analyzed by chi-square test. Continuous data were described as mean +/standard deviation and analyzed using standard t-test.

Significance was defined as P value less than 0.05 .

\section{Results}

There was a significant difference between the two groups as regard age, (P-value $<0.001)$, (Table 1 ). There was a significant difference between the two groups as regard Hypertension, Diabetes, and Mitral annular calcification. (P-value <0.001) However there was no significant difference as regard smoking, (Table 2). There was statistically high significant difference between the two groups as regard Gensini score and number of vessel affected. (P-value $<0.001$ ) (Table $3 \& 4$ ). There was statistically high significant difference between the two subgroups as regard Gensini score and number of vessel affected. (P-value < 0.001 ) (Table $5 \& 6$ ). There was a significant correlation between sum of aortic valve cusps thickness and (age - serum cholesterol level - Gensini score) (P-value <0.001) (Table 7) (Figures 2-8).

Table 1: Comparison between the two groups according to age.

\begin{tabular}{|c|c|c|c|c|}
\hline \multirow{2}{*}{ Groups } & \multicolumn{2}{|c|}{ Age (years) } & \multicolumn{2}{c|}{ T-test } \\
\cline { 2 - 5 } & Range & Mean \pm SD & t & P-value \\
\hline Group I & $37-61$ & $50.75 \pm 6.83$ & \multirow{2}{*}{7.088} & $<0.001^{* *}$ \\
\hline Group II & $39-65$ & $56.81 \pm 5.19$ & & \\
\hline
\end{tabular}

*Data are presented as mean \pm standard deviation.

Table 2: Comparison between the two groups according to other parameters.

\begin{tabular}{|c|c|c|c|c|c|c|c|c|}
\hline \multirow{2}{*}{} & \multicolumn{2}{|c|}{ Group I } & \multicolumn{2}{c|}{ Group II } & \multicolumn{2}{c|}{ Total } & \multicolumn{2}{c|}{ Chi-square } \\
\cline { 2 - 9 } & $\mathbf{N}$ & $\mathbf{\%}$ & $\mathbf{N}$ & $\mathbf{\%}$ & $\mathbf{N}$ & $\mathbf{\%}$ & $\mathbf{X}^{2}$ & P-value \\
\hline Smoking & 38 & 50.7 & 68 & 54.4 & 106 & 53 & 0.262 & 0.609 \\
\hline HTN & 24 & 32 & 83 & 66.4 & 107 & 53.5 & 22.297 & $<0.001^{* *}$ \\
\hline DM & 10 & 13.3 & 74 & 59.2 & 84 & 42 & 40.482 & $<0.001^{* *}$ \\
\hline $\begin{array}{c}\text { Serum } \\
\text { cholesterol } \\
\text { (mean) }\end{array}$ & 174.4 & 221.95 & & -- & & $<0.001^{* *}$ \\
\hline
\end{tabular}

* Data are presented as number of patients (group percentage)
Table 3: Comparison between the two groups according to Gensini score.

\begin{tabular}{|c|c|c|c|c|}
\hline \multirow{2}{*}{ Groups } & \multicolumn{2}{|c|}{ Gensini Score } & \multicolumn{2}{c|}{ T-test } \\
\cline { 2 - 5 } & Range & Mean \pm SD & t & P-value \\
\hline Group I & $0-84$ & $14.21 \pm 21.69$ & \multirow{2}{*}{6.368} & $<0.001^{* *}$ \\
\hline Group II & $0-168$ & $42.05 \pm 33.9$ & & \\
\hline
\end{tabular}

*Data are presented as mean \pm standard deviation.

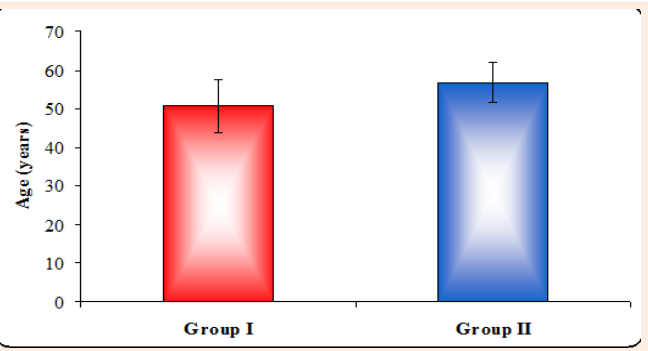

Figure 2: Comparison between the two groups according to age

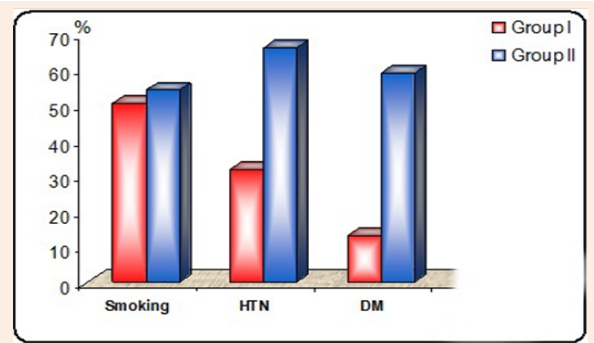

Figure 3: Comparison between the two groups according to other parameters.

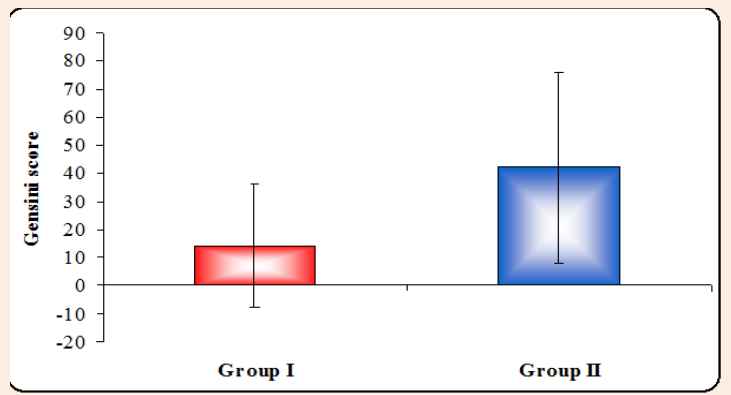

Figure 4: Comparison between the two groups according to Gensini score.

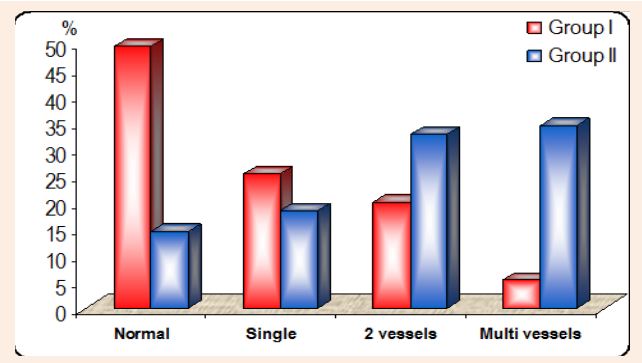

Figure 5: Comparison between the two groups according to number of vessels affected. 
Table 4: Comparison between the two groups according to number of vessels affected.

\begin{tabular}{|c|c|c|c|c|c|c|c|c|}
\hline \multirow{2}{*}{$\begin{array}{c}\text { Number } \\
\text { of Vessels } \\
\text { Affected }\end{array}$} & \multicolumn{9}{|c|}{ Groups } \\
\cline { 2 - 9 } & $\mathbf{2}$ & $\mathbf{9}$ & $\mathbf{N}$ & $\mathbf{\%}$ & $\mathbf{N}$ & $\mathbf{\%}$ & $\mathbf{X}^{2}$ & P-value \\
\hline Normal & 37 & 49.3 & 18 & 14.4 & 55 & 27.5 & 28.691 & $<0.001^{* *}$ \\
\hline CAD & 38 & 50.7 & 107 & 85.6 & 145 & 72.5 & & \\
\hline Single & 19 & 25.3 & 23 & 18.4 & 42 & 21 & 1.358 & 0.244 \\
\hline 2 vessels & 15 & 20 & 41 & 32.8 & 56 & 28 & 3.81 & $0.049^{*}$ \\
\hline $\begin{array}{c}\text { Multi } \\
\text { vessels }\end{array}$ & 4 & 5.3 & 43 & 34.4 & 47 & 23.5 & 22.029 & $<0.001^{* *}$ \\
\hline Total & 75 & 100 & 125 & 100 & 200 & 100 & & \\
\hline
\end{tabular}

* Data are presented as number of patients (group percentage).

Table 5: Comparison between the two subgroups according to Gensini score.

\begin{tabular}{|c|c|c|c|c|}
\hline \multirow{2}{*}{ Groups } & \multicolumn{2}{|c|}{ Gensini Score } & \multicolumn{2}{|c|}{ T-test } \\
\cline { 2 - 5 } & Range & Mean \pm SD & t & P-value \\
\hline Group IIa & $0-112$ & $32.74 \pm 28.51$ & \multirow{2}{*}{4.866} & $<0.001^{* *}$ \\
\hline Group IIb & $0-168$ & $61.83 \pm 36.25$ & & \\
\hline
\end{tabular}

*Data are presented as mean \pm standard deviation.

Table 6: Comparison between the two subgroups according to number of vessels affected.

\begin{tabular}{|c|c|c|c|c|c|c|c|c|}
\hline \multirow{3}{*}{$\begin{array}{c}\text { Number of } \\
\text { Vessels Affected }\end{array}$} & \multicolumn{8}{|c|}{ Subgroups } \\
\hline & \multicolumn{2}{|c|}{ Group IIa } & \multicolumn{2}{|c|}{ Group IIb } & \multicolumn{2}{|c|}{ Total } & \multicolumn{2}{|c|}{ Chi-square } \\
\hline & $\mathrm{N}$ & $\%$ & $\mathbf{N}$ & $\%$ & $\mathrm{~N}$ & $\%$ & $\mathrm{X}^{2}$ & P-value \\
\hline Normal & 16 & 18.8 & 2 & 5 & 18 & 14.4 & \multirow{2}{*}{4.217} & \multirow{2}{*}{$0.040^{*}$} \\
\hline CAD & 69 & 81.2 & 38 & 95 & 107 & 85.6 & & \\
\hline Single & 16 & 18.8 & 7 & 17.5 & 23 & 18.4 & 0.032 & 0.859 \\
\hline 2 vessels & 35 & 41.2 & 6 & 15 & 41 & 32.8 & 8.456 & $0.004^{*}$ \\
\hline Multi vessels & 18 & 21.2 & 25 & 62.5 & 43 & 34.4 & 20.583 & $<0.001^{* *}$ \\
\hline Total & 85 & 100 & 40 & 100 & 125 & 100 & & \\
\hline
\end{tabular}

Table 7: Correlation between sum of aortic valve cusps thickness and (age - serum cholesterol level - Gensini score).

\begin{tabular}{|c|c|c|}
\hline \multirow{2}{*}{} & \multicolumn{2}{|c|}{ Sum of Aortic Valve Cusps Thickness } \\
\cline { 2 - 3 } & $\mathbf{r}$ & P-value \\
\hline Age (years) & 0.389 & $<0.001^{* *}$ \\
\hline $\begin{array}{c}\text { Serum cholesterol } \\
\text { level }\end{array}$ & 0.495 & $<0.001^{* *}$ \\
\hline Gensini score & 0.605 & $<0.001^{* *}$ \\
\hline
\end{tabular}

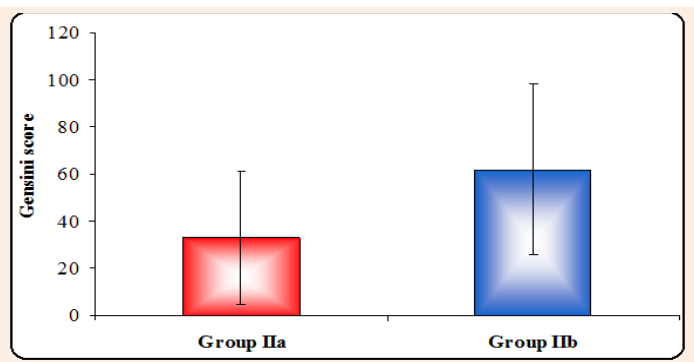

Figure 6: Comparison between the two subgroups according to Gensini score. 


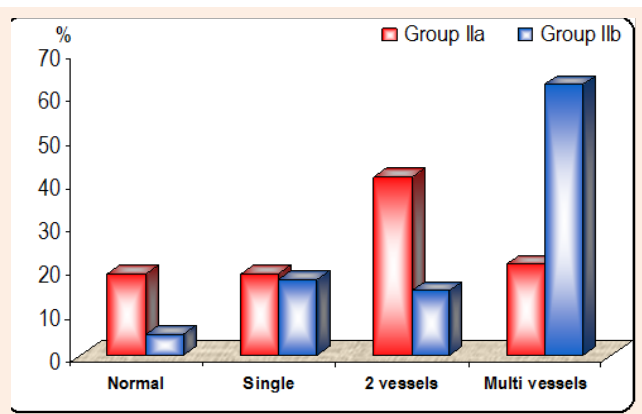

Figure 7: Comparison between the two subgroups according to number of vessels affected.

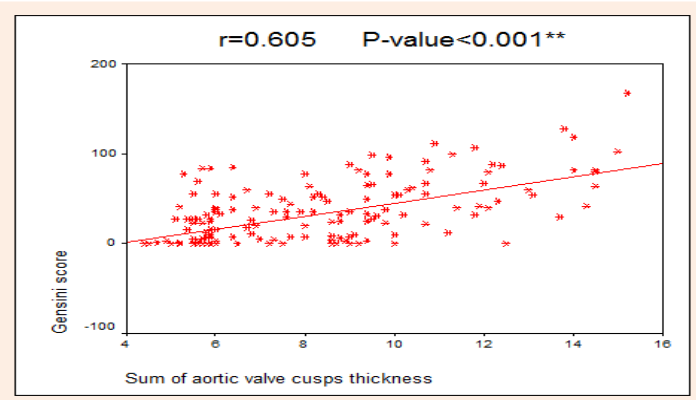

Figure 8: Correlation between sum of aortic valve cusps thickness and Gensini score.

\section{Discussion}

In the present study, Mean age and number of diabetic patients were significantly higher in the AVS group than the control group. These results are consistent with earlier reports showing a higher prevalence of traditional cardiovascular risk factors among patients with AVS [14]. Gensini score was found to be significantly higher in the sclerotic group $(42.05 \pm 33.9)$ compared to the normal group $(14.21 \pm 21.69)$ ( $p$ value $<0.001)$. Also, Gensini score was of significant difference between the two subgroups compared to the control group ( $p$ value $<0.001$ ). This finding is comparable with those of earlier studies assessing the relation between AVS and CAD.

In 2002, Kirsten and his colleagues studied the morphologic classification system for AVS by transesophageal echocardiography and correlated the subtypes of AVS with the presence of cardiovascular disease, they concluded that it is possible to identify a subgroup of patients with mixed nodular and diffuse sclerosis, were at increased risk for CAD including multivessel disease [15]. In 2006, Serdar et al. [16], studied the association between AVS and the extent of coronary atherosclerosis by means of the Gensini score system, he concluded that AVS is strongly interrelated with the coronary angiographic Gensini score. Echocardiographic detection of AVS in patients undergoing coronary angiography can predict the extent of coronary atherosclerosis [16].

Another study concluded that pathologic processes that may occur in coronary arteries may be identified more easily in the aortic valve and they suggest that once the diagnosis of AVS has been made by echocardiography, it should be considered a potential marker of CAD, and patients with diagnosed AVS should undergo intensive screening for CAD with aggressive management of modifiable risk factor reduction [14].

As regard number of affected coronary arteries, our study was in agreement with previous reports regarding the relationship between AVS and multivessels disease [17,19].

In our study of the 200 patients, coronary artery stenosis was found in 145 patients $(72.5 \%)$, of whom 107 were in the AVS group and 38 were in the non-AVS group. In the AVS group, CAD was found in all patients, of whom 23 (18.4\%) had singlevessel disease and 43 (34.4\%) had multivessels disease. In the non-AVS group, there were $50.7 \%$ CAD patients $(38 / 75)$, of whom $19(25.3 \%)$ had single-vessel disease and 4 (5.3\%) had multivessels disease. The AVS group had a higher positive rate of coronary angiography (85.6 vs. $50.7 \%$ ) and incidence rate of multivessels CAD (34.4 vs. 5.3\%) than the non-AVS group. This is in agreement with Shu-Jian et al. [18], study in which Of the 138 patients, significant coronary artery stenosis was found in 60 patients (43.5\%), of whom 37 were in the AVS group and 23 were in the non-AVS group. In the AVS group, CAD patients account for $63.8 \%(37 / 58)$, of whom $13(22.4 \%)$ had single-vessel disease and $24(41.4 \%)$ had multi-vessels disease. In the non-AVS group, there were $28.8 \%$ CAD patients (23/80), of whom $15(18.8 \%)$ had single-vessel disease and $8(10.0 \%)$ had multi-vessels disease. The AVS group had a higher positive rate of coronary angiography (63.8 vs. $28.8 \%$ ) and incidence rate of multi-vessels CAD (41.4 vs. $10.0 \%$ ) than the non-AVS group [18].

The association between AVS and increased coronary plaque burden may explain recent data regarding the increased risk of fatal cardiovascular causes and the risk of myocardial infarction in patients with AVS $[7,14]$. The reason for increased cardiovascular events associated with aortic sclerosis can be best explained by assuming that aortic sclerosis is an objective marker of CAD, as aortic sclerosis and CAD share many risk factors $[7,8]$.

As regard grading of AVS, Among the 125 patients with AVS, 85 (68\%) had grade II AVS (group IIa) and 40(32\%) had grade III and IV AVS (group IIb). There was statistically significant difference between the two subgroups as regard the extent of CAD, This disagree with study provided by Kirsten Tolstrup et al. who reported that Among the 66 patients with AVS, 54 (82\%) had grade II AVS and 12 (18\%) had grade III and IV AVS with no significant association between the grading of AVS and CAD [15].

\section{Conclusion}

A. AVS is strongly correlated with the extent of coronary artery disease

B. Echocardiographic detection of AVS in patients undergoing coronary angiography may be applied as a new surrogate marker of the extent of coronary atherosclerosis.

C. AVS may predict the extent of CAD.

D. Extent of AVS is highly correlated with the extent of CAD.

E. AVS is associated with atherosclerosis risk factors like age, dyslipidemia and diabetes mellitus. 


\section{References}

1. Boon A, Cheriex E, Lodder J, Kessels F (1997) Cardiac valve calcification: characteristics of patients with calcification of the mitral annulus or aortic valve. Heart 78(5): 472-474.

2. Lindroos M, Kupari M, Heikkila J, Tilvis R (1993) Prevalence of aortic valve abnormalities in the elderly: an echocardiographic study of a random population sample. J Am Coll Cardiol 21(5): 1220-1225.

3. Tolstrup K, Crawford MH, Roldan CA (2002) Morphologic characteristics of aortic valve sclerosis by transesophageal echocardiography: importance for the prediction of coronary artery disease. Cardiology 98(3): 154-158.

4. Stewart BF, Siscovick D, Lind BK, Gardin JM, Gottdiener JS, et al (1997) Clinical factors associated calcific aortic valve disease. J Am Coll Cardiol 29(3): 630-634

5. Agmon Y, Khandheria BK, Meissner I, Sicks JR, O’Fallon WM, et al. (2001) Aortic valve sclerosis and atherosclerosis: different manifestations of the same disease? Insights from a populationbased study. J Am Coll Cardiol 38(3): 827-834.

6. Adler Y, Herz I, Vaturi M, Fusman R, Shohat-Zabarski R, et al. (1998) Mitral annular calcium detected by transthoracic echocardiography is a marker for high prevalence and severity of coronary artery disease in patients undergoing coronary angiography. Am J Cardiol 82(10): 1183-1186

7. Otto CM, Lind BK, Kitzman DW, Gersh BJ, Siscovick P, et al. (1999) Association of aortic- valve sclerosis with cardiovascular mortality and morbidity in the elderly. N Engl J Med 341(3): 142-147.

8. Carabello RA (1999) Aortic sclerosis - a window to the coronary arteries? N Engl J Med 341(3): 193-195.

9. Hsu SY, Hsieh IC, Chang SH, Wen MS, Hung KC (2005) Aortic valve sclerosis is an echocardiographic indicator of significant coronary disease in patients undergoing diagnostic coronary angiography. Int J Clin Pract 59(1): 72-77.
10. (1999) ACC/AHA Guidelines for Coronary Angiography: Executive Summary and Recommendations A Report of the American College of Cardiology/American Heart Association Task Force on Practice Guidelines (Committee on Coronary Angiography) Developed in collaboration with the Society for Cardiac Angiography and Interventions. Circulation 99(17): 2345-2357.

11. ACC/AHA/AAPA/ABC/ACPM/AGS/APhA/ASH/ASPC/NMA/PCNA Guideline for the Prevention, Detection, Evaluation, and Management of High Blood Pressure in Adults 2017.

12. Roberto M Lang, Michelle Bierig, Richard B Devereux (2005) Recommendations for chamber quantification. Eur J Echocardiography 79: 108.

13. Gensini GG (1983) A more meaningful scoring system for determining the severity of coronary heart disease. Am J Cardiol 51(3): 606

14. Prasad Y, Bhalodkar NC (2004) Aortic sclerosis - a marker of coronary atherosclerosis. Clin Cardiol 27(12): 671-673.

15. Tolstrup K, Crawford M, Roldan CA (2002) Morphologic Characteristics of Aortic Valve Sclerosis by Transesophageal Echocardiography: Importance for the Prediction of Coronary Artery Disease. Cardiology 98(3): 154-158.

16. Soydinc S, Davutoglu V, Dundar A, Aksoy M (2006) Relationship between Aortic Valve Sclerosis and the Extent of Coronary Artery Disease in Patients Undergoing Diagnostic Coronary Angiography. Cardiology 106(4): 277-282.

17. Adler Y, Vaturi M, Herz I, Iakobishvili Z, Toaf J, et al. (2002) Nonobstructive aortic valve calcification: a window to significant coronary artery disease. Atherosclerosis 161(1): 193-197.

18. Shu-Jian Sui, Man-Yi Ren, Fu-Yu Xu, Yun Zhang (2007) A High Association of Aortic Valve Sclerosis Detected by Transthoracic Echocardiography with Coronary Arteriosclerosis. Cardiology 108(4): 322-330. 